\title{
Hybrid-functional electronic structure of multilayer graphene
}

\author{
Marco Campetella, ${ }^{1, *}$ Jacopo Baima $\odot,{ }^{1}$ Nguyen Minh Nguyen $\odot,{ }^{2}$ Lorenzo Maschio, ${ }^{3}$ \\ Francesco Mauri, ${ }^{4}$ and Matteo Calandra ${ }^{5,1, \dagger}$ \\ ${ }^{1}$ Sorbonne Université, CNRS, Institut des Nanosciences de Paris, UMR7588, F-75252, Paris, France \\ ${ }^{2}$ International Research Centre MagTop, Institute of Physics, Polish Academy of Sciences, Aleja Lotnikow 32/46, PL-02668 Warsaw, Poland \\ ${ }^{3}$ Dipartimento di Chimica and Centre of Excellence NIS (Nanostructured Interfaces and Surfaces), Università di Torino, \\ via P. Giuria 5, I-10125 Turin, Italy \\ ${ }^{4}$ Dipartimento di Fisica, Università di Roma La Sapienza, Piazzale Aldo Moro 5, I-00185 Roma, Italy \\ ${ }^{5}$ Department of Physics, University of Trento, Via Sommarive 14, 38123 Povo, Italy
}

(Received 4 January 2020; accepted 24 March 2020; published 30 April 2020)

\begin{abstract}
Multilayer graphene with rhombohedral and Bernal stacking is supposed to be metallic, as predicted by density functional theory calculations using semilocal functionals. However, recent angular resolved photoemission and transport data have questioned this point of view. In particular, rhombohedral flakes are suggested to be magnetic insulators, a view supported also by hybrid-functional calculations. Bernal flakes composed of an even number of layers are insulating (for $N \leqslant 6$ ), while those composed of an odd number of layers are pseudogapped (for $N \leqslant 7$ ). Here, by systematically benchmarking with plane-waves codes, we develop very accurate all-electron Gaussian basis sets for graphene multilayers, allowing a precise description of the electronic structure in the $100 \mathrm{meV}$ energy range from the Fermi energy at the hybrid-functional level. We find, in agreement with our previous calculations, that rhombohedral stacked multilayers are gapped and magnetic. However, the valence band curvature and the details of the electronic structure at the $\sim 10 \mathrm{meV}$ scale show a dependence on the basis set. A substantially extended basis set is needed to describe the long-range interlayer interactions and, consequently, to correctly reproduce the effective mass of the valence band top at the $K$ point. In the case of Bernal stacking, we show that exact exchange gaps the flakes composed by four layers and opens pseudogaps for $N=3,6,7,8$. However, the gap or pseudogap size and its behavior as a function of thickness are not compatible with experimental data. Moreover, hybrid functionals lead to a metallic solution for five layers and a magnetic ground state for five, six, and eight layers. Magnetism is very weak with practically no effect on the electronic structure and the magnetic moments are mostly concentrated in the central layers. Our hybridfunctional calculations on trilayer Bernal graphene are in excellent agreement with $G W$ results. For thicker multilayers, our calculations are a benchmark for many-body theoretical modeling of the low energy electronic structure.
\end{abstract}

DOI: 10.1103/PhysRevB.101.165437

\section{INTRODUCTION}

The electronic structure of multilayer graphene has been calculated with a variety of techniques such as tight binding with parameters fitted on experiments [1-4], the effective mass approximation or low energy expansions [5,6], density functional theory with semilocal functionals (typically LDA or PBE) [7,8], and nonmagnetic RPA [9] (bilayer graphene) and $G W$ approximations $[9,10]$ (bilayer and trilayer graphene). The view emerging from all these calculations is that all systems are metallic/semimetallic (i.e., they do not have a gap). In more details, close to the Fermi level, at the special point $K$ of the Brillouin zone, rhombohedral stacked multilayers display a flat surface state. Bernal stacked multilayers show metallic massive bands for even $N$, while a Dirac cone coexists with massive bands for odd $N$ (see Fig. 1 for the $N=3,4$ case).

\footnotetext{
*marco.campetella@upmc.fr

${ }^{\dagger}$ matteo.calandra@upmc.fr
}

Several experiments contradict this view. Transport measurements on unsupported rhombohedral flakes composed of three and four layers [11,12] show the presence of an insulating state with gaps larger than $40 \mathrm{meV}$. Recent magnetotransport experiments on unsupported $\mathrm{ABC}$ trilayers display large and field-effect tunable magnetoconductance hysteresis, suggestive of a magnetic state [13]. At larger $n$ doping, the magnetic state is predicted to melt in an half-metallic ground state [14]. Two layers of bilayer graphene twisted by tiny angles have been shown to form uniform four-layer ABCA graphene regions with a $9.5 \mathrm{meV}$ gap as measured in STM and attributed to many-body effects [15].

Samples of rhombohedral stacked graphene with thickness up to 50 layers were recently isolated [16-18]. The rhombohedral stacking was identified via Raman spectroscopy $[16,19]$ and Landau level measurements $[16,18]$.

ARPES data on 14 layers samples were found to be consistent with the occurrence of a magnetic state [17], by comparison of the valence band effective mass at the $K$ point with hybrid-functional calculations for magnetic and nonmagnetic solutions $[17,20]$. The curvature of the top of 

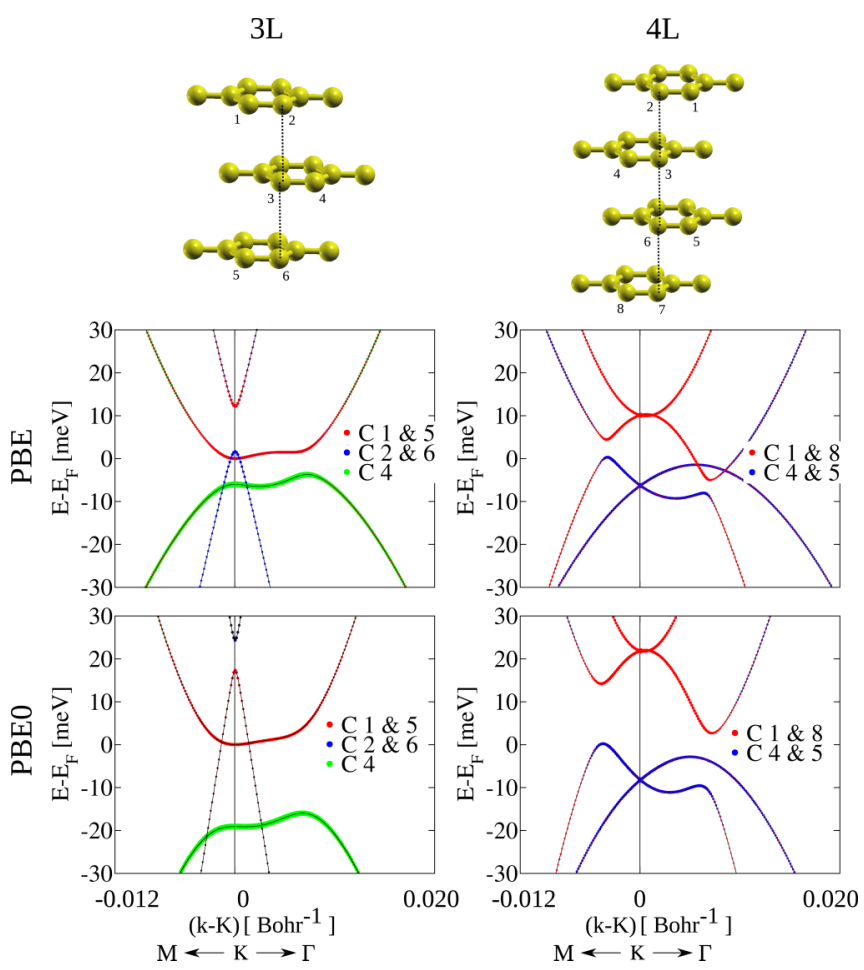

FIG. 1. Top panels: Atom numbering scheme for three- and fourlayer Bernal graphene. Bottom panels: PBE and PBE0 electronic structure of trilayer (left panels) and four-layer (right panels) Bernal graphene. The color is proportional to the $p_{z}$ orbital character of the carbon atoms. The electronic bands are plotted around the $\mathbf{K}$ point that has been chosen as origin.

the valence bands at $K$ was found to be much larger in the magnetic case than in the nonmagnetic case.

The situation is similar for Bernal stacking, as several measurements suggest the occurrence of a gapped state on suspended samples [21-24]. Among them, a very recent paper [23] show that all $N$-layer Bernal suspended graphene flakes with $2 \leqslant N \leqslant 7$ are insulating or pseudogapped. Specifically, the resistance at the charge neutrality point of suspended flakes with $N=2,4,6$ is in the range $5 \times 10^{3}$ to $5 \times 10^{5} \mathrm{k} \Omega$ at $T=0.25 \mathrm{~K}$, monotonically increasing with thickness. In the odd number of layer case, the resistance at charge neutrality is smaller and of the order of 25 to $55 \mathrm{k} \Omega$ at $T=0.25 \mathrm{~K}$, monotonically decreasing with thickness. The behavior of flakes with an odd number of layers is more suggestive of a pseudogapped phase than that of a completely insulating state. For even $N$ the gap measured via transport increases with thickness and ranges between 1 and $13 \mathrm{meV}$, substantially smaller than for the case of $\mathrm{ABC}$ multilayers.

In Bernal graphene multilayers, the massive bands close to the Fermi level have small Fermi velocities and large effective masses. Given the small kinetic energy of the electrons in these bands, a gap could open due to electron-electron interaction effects not included in semilocal functionals, as it happens for ABC graphene multilayers [20]. Carbon based systems hosting correlated states are not so uncommon, as this is actually proposed to happen in graphene multilayers with rhombohedral $(\mathrm{ABC})$ stacking $[11,12,16,17,20]$,

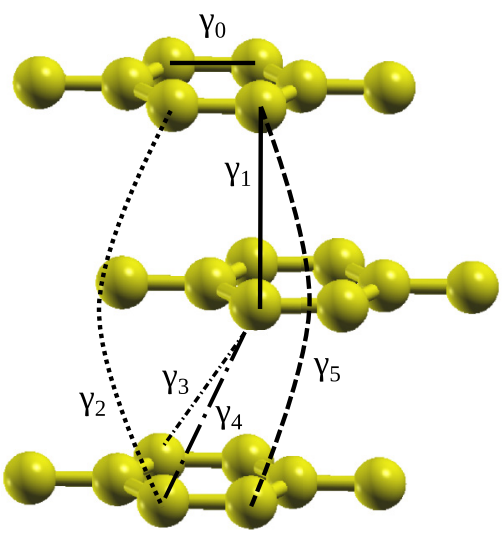

FIG. 2. Tight-binding hopping parameters for multilayer graphene with Bernal stacking.

in twisted bilayer graphene [25,26], or in diamond(111) [27]. In all these cases, the correlated state is proposed to emerge from flat bands. From the theoretical point of view, a self-consistent tight-binding calculation [28] with empirical inclusion of Hartree and exchange terms on Bernal multilayers suggests that the exchange interaction substantially modifies the electronic structure, via the renormalization of the $\gamma_{2}$ hopping term (see Fig. 2 for the hopping processes). However, the possible occurrence of magnetism was not studied in this work, and the empirical form of the electron-electron interaction calls for more accurate calculations.

A first step towards the understanding of the electronelectron interaction effects in multilayer graphene is, then, the determination of the role of the exchange interaction. This can be quantitatively evaluated at the mean-field level by using hybrid functionals including a certain percentage of HartreeFock exchange. In single layer graphene the renormalization of the Fermi velocity is well captured by hybrid functionals [29]. The difficulty is, however, that hybrid-functional calculations are computationally demanding, particularly in the case of multilayer graphene with Bernal stacking as the Fermi surface is very narrow (linear dimension of $\approx 0.01 \AA^{-1}$ ) and the Brillouin zone sampling becomes soon prohibitive, particularly if plane-wave codes are used. This difficulty has hindered, up to now, calculations beyond semilocal functionals in these systems.

Here, by developing a very accurate basis set tailored for multilayer graphene, we perform all-electron electronic structure calculations with the inclusion of exact exchange and ultradense Brillouin zone sampling (up to $1200 \times 1200$ for the self-consistent calculation and up to $12000 \times 12000$ for the density of states). We carry out an in-depth analysis of the electronic structure and compare our results with experimental data. We consider (6 and 14 layers) both the case of thick $\mathrm{ABC}$ graphene samples and Bernal stacked flakes up to seven layers.

The paper is organized as follows. In Sec. II we describe the technical details of the calculation. In Sec. IV we analyze the possible stabilization of magnetic state in multilayer Bernal graphene. Finally we discuss the electronic structure of these systems. 


\section{COMPUTATIONAL DETAILS}

\section{A. Simulation parameters}

All-electron electronic structure calculations were performed using the CRYSTAL code [30]. The PBE and the PBE0 [31] hybrid functionals have been used for DFT calculations with an ad hoc optimized def2-TZVP Gaussiantype basis sets [32] for the $\mathrm{C}$ atoms labeled as TZVP+ (see Sec. II B for more details) for Bernal graphene and a QZVP adapted basis set for rhombohedral graphene. The Gaussian exponents and coefficients are reported in the Supplemental Material (SM) [33]. In order to avoid numerical instabilities due to an ill-conditioned overlap matrix (basis set near-linear dependency), we removed the eigenvectors belonging to the smallest eigenvalues with a threshold of $10^{-5}$. The band flatness and the extreme localization of the low-energy states around the special point $\mathbf{K}$ require an ultradense sampling with an electronic $k$ mesh of $1200 \times 1200$. The real space integration tolerances was set to 1111111540 (in order to use such extended basis set) and with an energy tolerance of $10^{-11} \mathrm{Ha}$ for the total energy convergence. The density of states (DOS) is obtained with a Gaussian smearing of $0.00001 \mathrm{Ha}$. The grid points on which the DOS has been calculated is characterized by a square grid centered on the $\mathbf{K}$ point. The square edge extension is $3 / 10$ of the length of the reciprocal space basis vector, and the total number of $k$ points used is 641601 . It is equivalent to a $k$ mesh of $12000 \times 12000$ on the entire BZ. Such a dense grid was needed to converge the DOS. In the case of magnetic calculations, we fix the magnetic state in the first iteration of the self-consistent cycle, and then we release the constraint. We worked in fixed geometry and we have chosen an in-plane lattice parameter of $a=2.461 \AA$ and an interplane distance of $3.347 \AA$. The distance between two adjacent periodic images is $13.38 \AA$ along the $z$ direction. Moreover, for the trilayer and quadrilayer Bernal graphene, density functional theory calculations with the PBE functional are performed using the Quantum-Espresso [34,35] code as well, in order to verify the consistency between pseudopotential and Gaussian basis set. In this case, for carbon we use norm conserving and PAW pseudopotentials. We use an energy cutoff up to 65 Ry for all calculations. For the exchange correlation energy we adopt the generalized gradient approximation (GGA). The charge density integration over the Brillouin zone (BZ) is performed using an uniform $512 \times 512$ Monkhorst and Pack grid [36]. The geometry of the systems is the same used for CRYSTAL. In PBE, the electronic structures are consistent within 2-3 meV and do not present qualitative differences. It is, however, difficult to infer if the residual differences come from the fact of using a Gaussian basis set instead of plane waves or if it is due to the use of an all-electron calculation against a pseudopotential one.

\section{B. Accuracy of Gaussian basis set}

The choice of a suitable basis set (BS) is a critical approximation for the description of very low energy electronic structure of multilayer graphene. As mentioned above, we used atom-centered Hermite-Gaussian functions, which mimic atomic orbitals. In general, these kinds of BSs require a lower number of basis set functions to provide good results and allow efficient computation of exact exchange integrals, but the quality of the approximation is more difficult to control with respect to plane-wave BSs, and may be material dependent. This is especially true for (semi)metallic solids, for which fewer basis sets have been devised and tested.

Among all-electron Gaussian BSs optimized from solid state simulations, a popular choice which normally results in good accuracy is to use the POB-TZVP BSs [37] which some of us have recently used for the study of ABC-stacked graphene multilayers $[14,17,20]$. In order to validate its reliability for the present study, we have compared the band structures of the graphene multilayers at the PBE level of theory with those obtained by means of the plane-wave calculations. As already mentioned, the convergence of the latter method with BS size is easier to verify, and will therefore be taken as reference. The results are reported in Fig. 3. As it can be seen, the POB-TZVP basis set does not exactly recover the results of the plane waves for Bernal multilayers. For instance, if we consider the odd-numbered layers systems, the two Dirac cones are located in an erroneous position. The bottom Dirac cone is always below the Fermi level, and in some cases it drops down to energy lower than $-30 \mathrm{meV}$, in this way it results absent in the energy region used in Fig. 3 (see 5L and 7L). As discussed in Sec. III A 2, the position of the Dirac cone is determined by long-distance out-of-plane interactions beyond the first neighboring layer. These kinds of interactions are difficult to describe with a local basis without the inclusion of diffuse functions. As a consequence, this basis set results in incorrect populations of both electrons and holes. The band dispersion for the even-numbered layers systems is incorrect as well. For this reason we have developed a new basis set for the $\mathrm{C}$ atom. We started from the def2-TZVP BS [32] that has three more Gaussian functions in comparison to the POBTZVP one: one $s$, one $d$, and one $f$. It can be freely downloaded from the basis set exchange site [38]. Since this basis set is devised for molecules, we have optimized the exponents $(\alpha)$ of the most diffused Gaussian functions, namely those with $\alpha<0.3$, minimizing the energy. The resulting basis set is named TZVP+ [39] for simplicity. The computed bands structure for TZVP+ is reported in Fig. 3. It can be seen that, in this case, the bands obtained by means of the Gaussian basis set reproduces all the main features of the plane-wave band structures. TZVP+, definitely, describes in a satisfactory way the interactions that are present in these systems. For the sake of testing the reliability of the TZVP+ basis set again, we have carried out the same calculation using a larger QZV(P) basis, which we have also reoptimized starting from a def2-QZVP molecular BS, but where we have reduced the high angular momentum polarization functions to the ones of the TZVP+ basis. As shown in Fig. 3 the differences between the two basis sets, around the Fermi level, are very small. Hence, because of the QZV $(\mathrm{P})$ high computational cost, we will use the TZVP+ basis set in our calculation.

In contrast, in the case of ABC-stacked multilayers the POB-TZVP basis produces much more satisfactory results, as there are no qualitative errors (e.g., band occupations) which appear for the AB stacking. Figure 4 shows PBE band structures for 6 layer and 14 layer. However, the curvature of the nearly flat band at the Fermi level is underestimated by this Gaussian BS. The curvature, and thus the BS error, increases 
$\mathrm{QE} / \mathrm{PBE}$
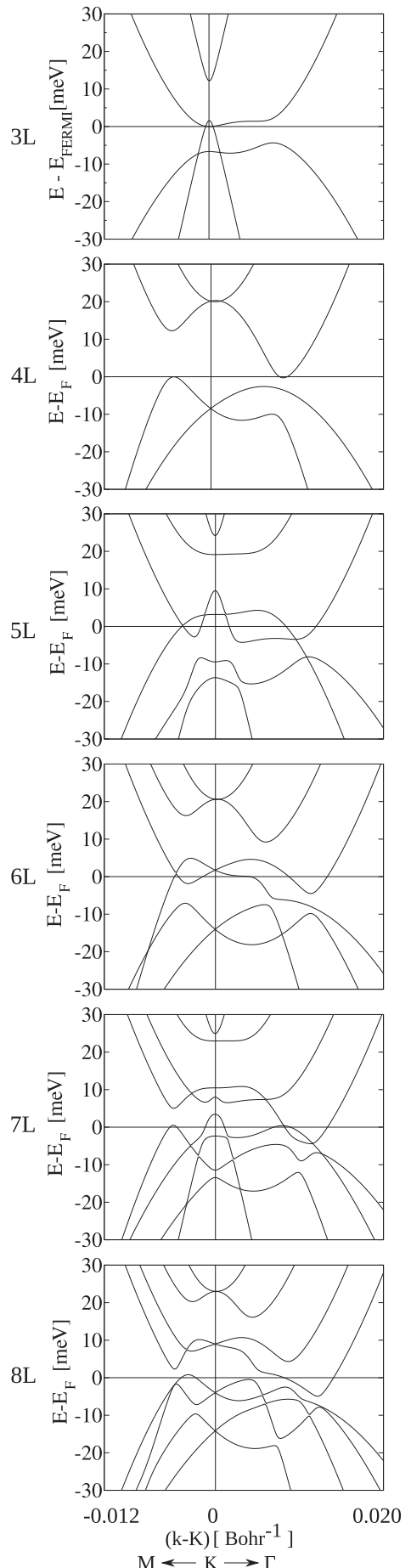

CRY / PBE / TZVP+
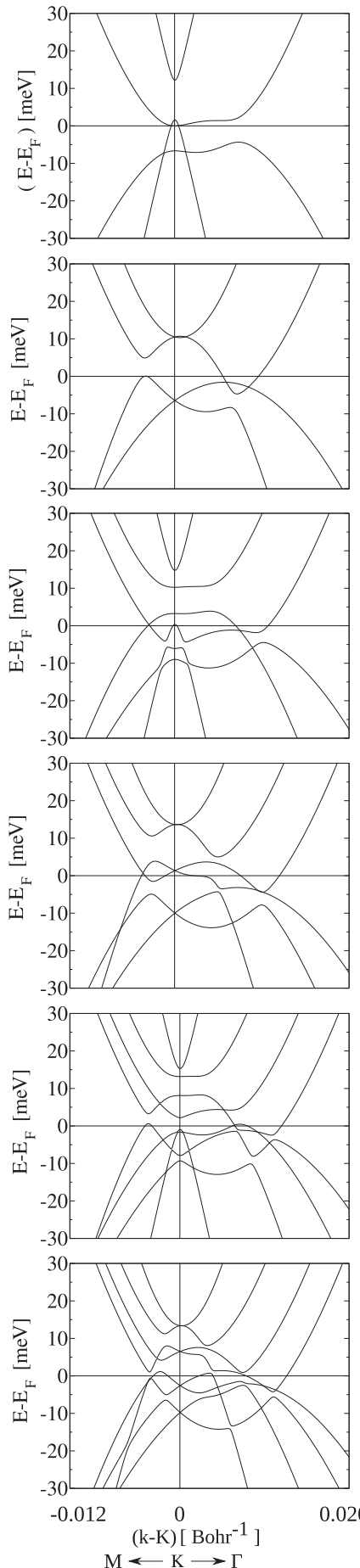

CRY / PBE / TVZP
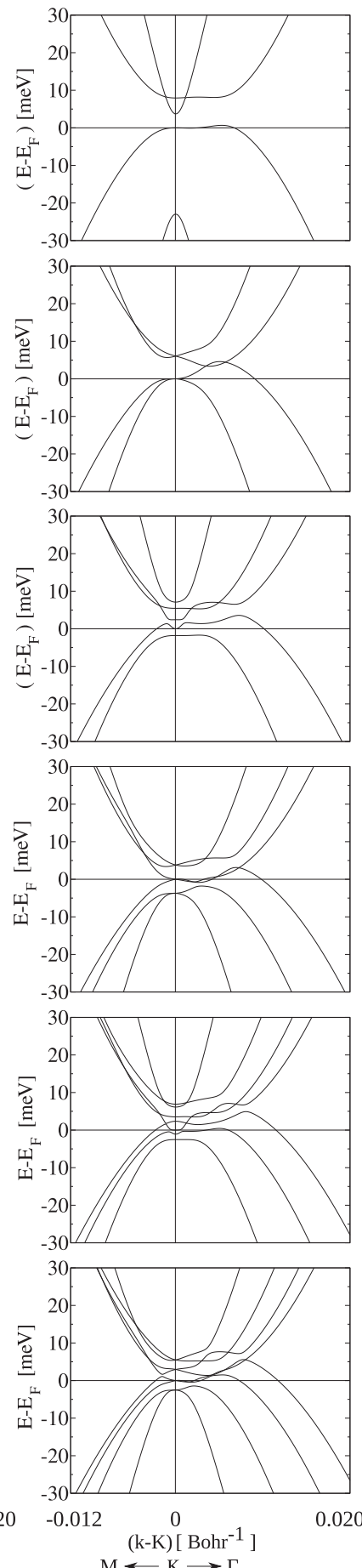

CRY / PBE / QZVP
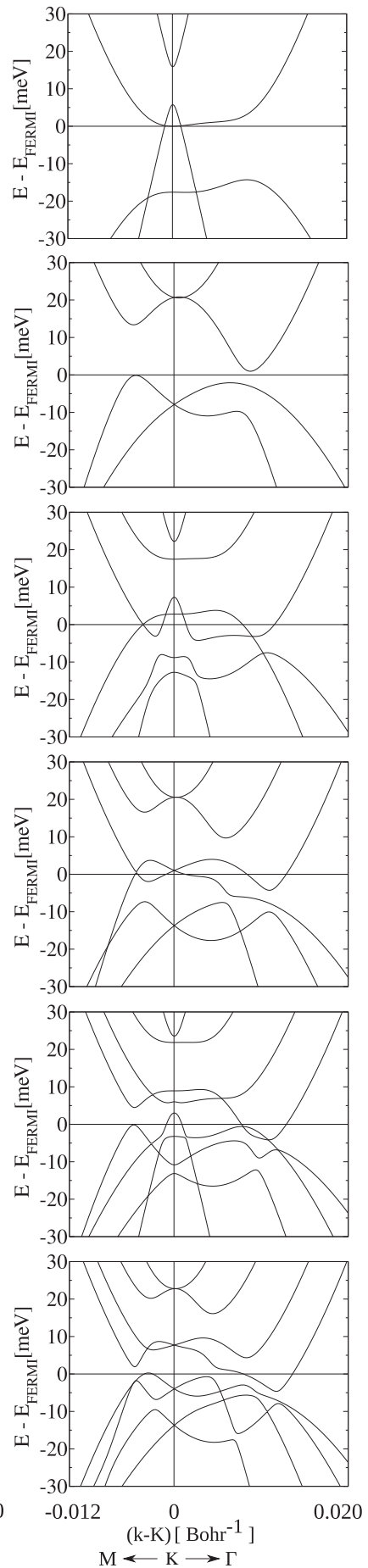

FIG. 3. PBE band structure of all studied multilayer systems with Bernal stacking. On the left are reported the calculations carried out with QE, while on the other columns the calculations made exploiting the CRYSTAL code, using different basis sets. From left to right we have the TVZP+ basis set, the standard TVZP basis set, and finally the QZVP basis set.

with the number of layers, suggesting that also in this case the problem lies in the description of long-range out-of-plane interactions. Conversely, the TZVP+ basis overestimates the curvature by roughly the same amount. In order to obtain full agreement with the plane-wave calculations, we have to use a larger QZV (P) basis.
We report here also the PBE0 result for 14 layers (see Fig. 5), for which the larger discrepancies between BSs are observed. Using the most accurate QZV(P) basis, we also obtain an enhanced curvature of the nearly flat band with respect to the POB-TZVP results, with a bandwidth of $8 \mathrm{meV}$ as opposed to less than $2 \mathrm{meV}$ reported in previous 
$\mathrm{QE} / \mathrm{PBE}$

CRY / PBE / TZVP

CRY / PBE / TZVP+

$\mathrm{CRY} / \mathrm{PBE} / \mathrm{QZV}(\mathrm{P})$
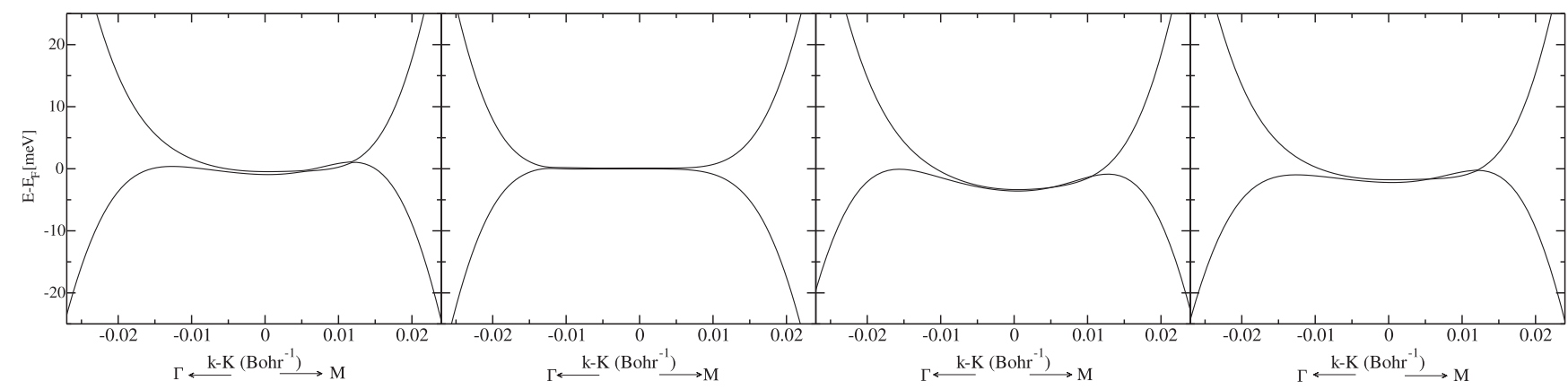

$\mathrm{QE} / \mathrm{PBE}$

CRY / PBE / TZVP

$\mathrm{CRY} / \mathrm{PBE} / \mathrm{TZVP}+$

CRY / PBE / QZV(P)

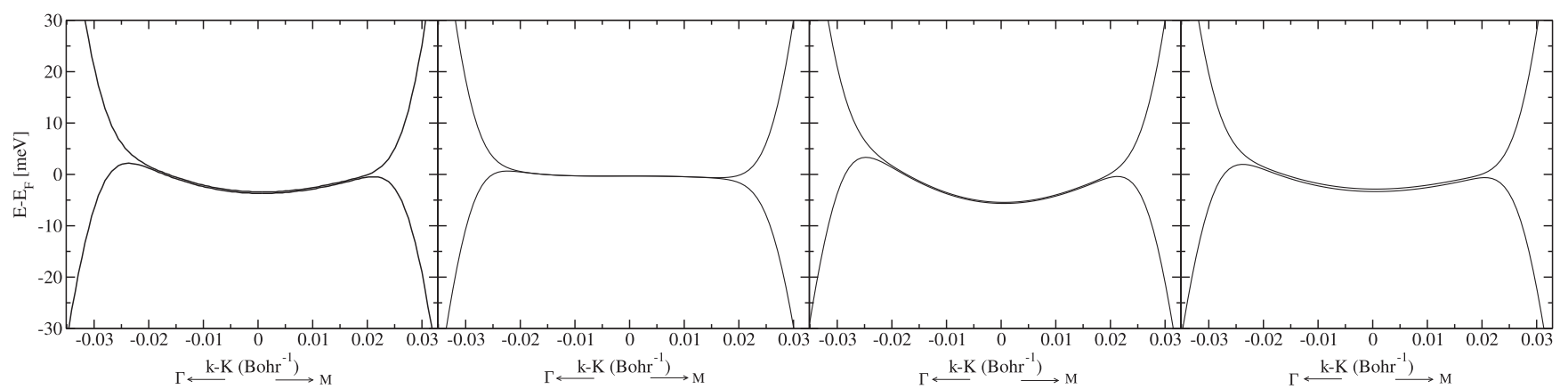

FIG. 4. PBE band structures for rhombohedral stacked multilayers (top: 6 layers, bottom: 14 layers) obtained with different approaches. On the left are reported the calculation carried out with QE, while the other calculations are made using the CRYSTAL code (the basis set specified in the figure, of increasing size from left to right).

publications [17], which are however unaffected in the qualitative results and conclusions.

\section{RESULTS FOR BERNAL MULTILAYERS}

\section{A. Bands decomposition}

\section{Structure of multilayer Bernal graphene}

In Fig. 1 (top panels) we report the crystal structures and atom labeling for $N=3,4$ multilayer graphene. It is important to note that in the case of Bernal stacking, the two inequivalent atoms per layer have different connectivity to out-of-plane nearest neighbors. One of the two atoms in each layer is vertically connected to two out-of-plane nearest neighbor atoms in nearby bilayers, i.e., it has one neighbor exactly on top and one exactly below. We label this atom to be of the A type. The other atom in the layer has no out-of-plane neighbors in nearest layers, neither on top nor below, and we label it as being of the B type [40,41]. A and B type atoms alternate within each layer, however A atoms lay always exactly on top or below A atoms in other neighboring layers. The out-of-plane hopping between nearest neighbors A atoms is normally labeled $\gamma_{1}$.

\section{Trilayer graphene}

The PBE electronic structure of trilayer graphene close to the Fermi level is composed of three bands: a gapped and slightly hole-doped Dirac cone and two gapped massive bands, as shown in Fig. 1. The band overlap between the hole doped part of the gapped Dirac cone and the empty massive band is approximately $1.8 \mathrm{meV}$. The bonding part of the gapped Dirac cone is formed mainly by $2 p_{z}$ orbitals of one of the two carbon atoms in the two outermost layers, namely atoms 2 and 6 . These atoms are of the A type. The nonbonding part of the gapped Dirac cone as well as the empty massive bands are, on the contrary, mostly formed by $2 p_{z}$ orbitals of B-type carbon atoms in the outermost layers, atoms 1 and 5. Finally, the occupied massive band is mostly formed by atom

\section{CRY / PBE0 / QZV(P)}

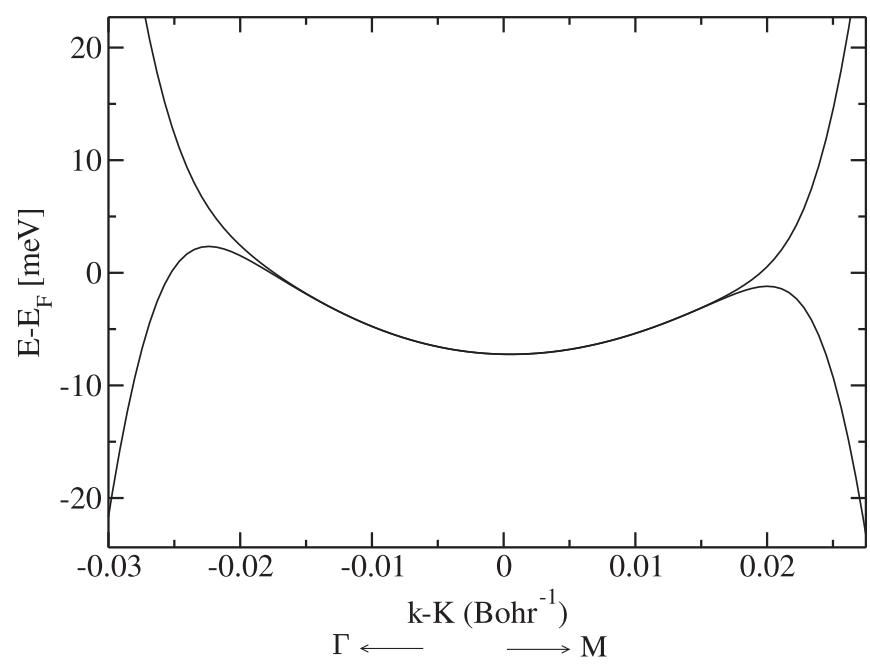

FIG. 5. PBE0 band structure for rhombohedral stacked 14 layer, obtained with the QZV $(\mathrm{P})$ basis (the most accurate basis set size). 

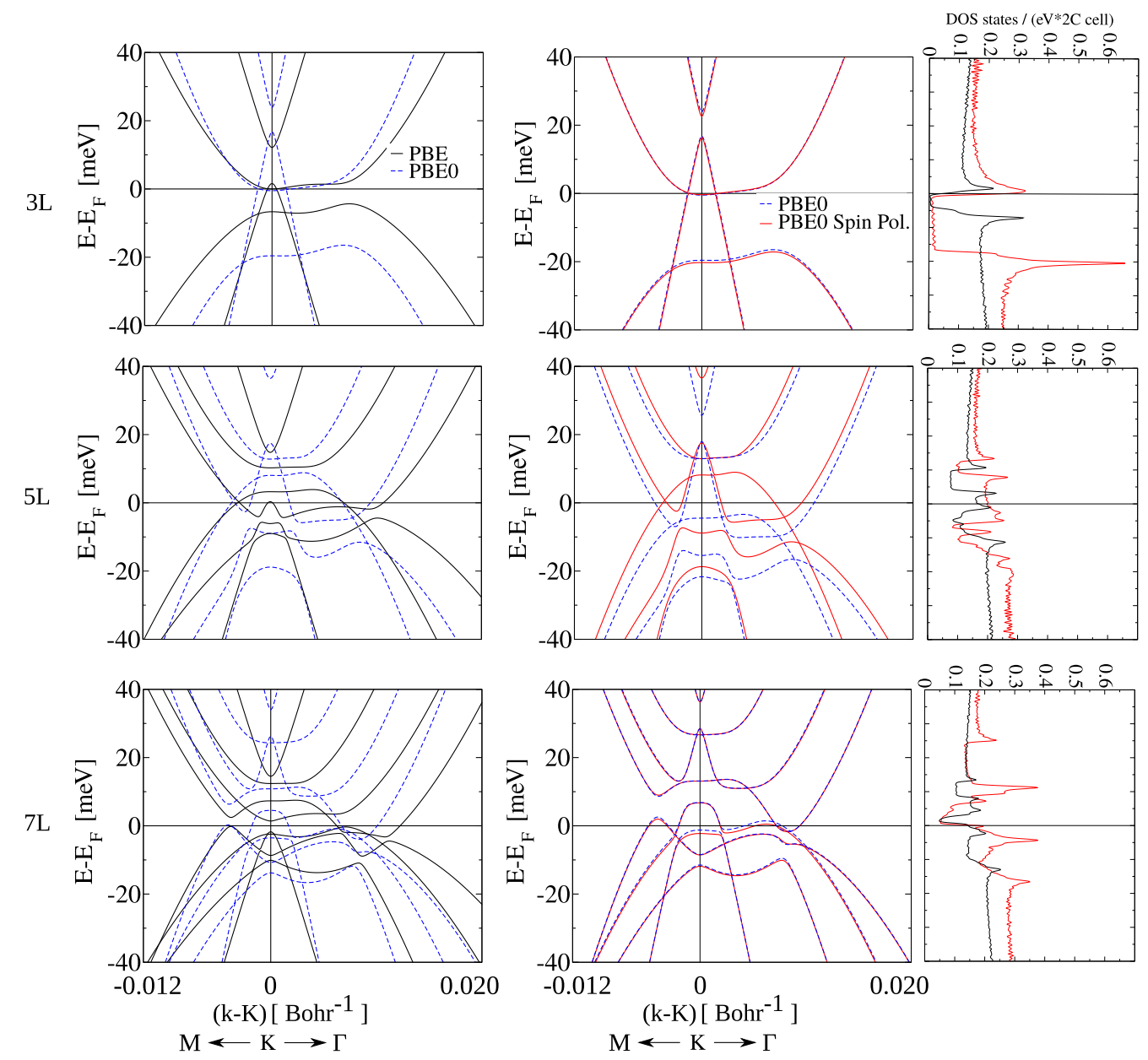

FIG. 6. Left panels: PBE versus PBE0 electronic structure for Bernal graphene multilayers with $N=3,5$, 7. Middle panels: PBE0 versus PBE0 spin-polarized electronic structures. The PBE0 magnetic and PBE nonmagnetic density of states are reported in the rightmost panels.

4 in the innermost layer, that is of B type. Atom 3 (A type) does not contribute to the electronic structure in this energy region. Thus, all massive bands are mainly due to $\mathrm{B}$ atoms (i.e., atoms having no neighboring atoms exactly on top and thus not connected by the $\gamma_{1}$ hopping). The electronic spectrum is not particle hole symmetric, mainly because the gapped Dirac cone is shifted with respect to the center of the massive bands. However, even the massive bands themselves are not particle-hole symmetric with respect to the other. In a minimal tight-binding model in which only the $\gamma_{0}$ (in-plane nearest neighbors hopping) and $\gamma_{1}$ (first out-of-plane neighbor) are considered, the spectrum is completely particle hole symmetric. As additional in-plane hoppings do not shift in energy the Dirac cone, but simply change the slope of the Dirac bands, it follows that the position of the Dirac point with respect to the massive bands can only be determined by long-distance out-of-plane hoppings. Previous work [28] suggested that this could be due to the $\gamma_{2}$ hopping, namely the hopping between atoms 1 and 5, i.e., the vertical hopping process between two B-type atoms laying in next nearest neighbor layers. This hopping is, of course, relevant only for $N \geqslant 3$. Moreover, we can also note that, even if the trilayer is not gapped in PBE, the large Fermi velocity of the Dirac cone leads to a small density of states at the Fermi level and the appearance of a pseudogap of $\approx 3.5 \mathrm{meV}$, as it can be seen in Fig. 6 .
PBE0 exhibits a similar electronic structure, as a matter of fact we can retrieve the gapped Dirac cone and the two massive bands as well. However, in this case, the exact exchange in the exchange-correlation energy has slightly changed the atomic contributions to the electronic bands. As in the previous case, the main character of the massive bands comes from B type. However, for what concerns the Dirac cone, now the bonding part is due to atoms 1 and 5 (B type) while the nonbonding part is due to atoms 2 and 6 (A type), the opposite of the PBE case. The Dirac cones are moved to higher energies, as compared to PBE, and the gap between them decreases from 10 (PBE) to $8 \mathrm{meV}$ (PBE0).

It is worthwhile to stress that our PBE0 calculation is in excellent agreement with the $G W$ calculation of Ref. [10].

\section{Four-layer graphene}

The low-energy electronic structure of four-layer graphene is composed of four massive bands. There is a small band overlap between the valence and conduction bands so that the system is metallic in PBE, as shown in Figs. 1 and 7. All bands in a $30 \mathrm{meV}$ energy window from the Fermi level are formed by the $2 p_{z}$ states of $\mathrm{B}$ atoms (i.e., not connected by $\gamma_{1}$ ). More specifically, the bands are formed by the $2 p_{z}$ states of atoms 1 and 8 in the outer layers, while the occupied bands are formed 

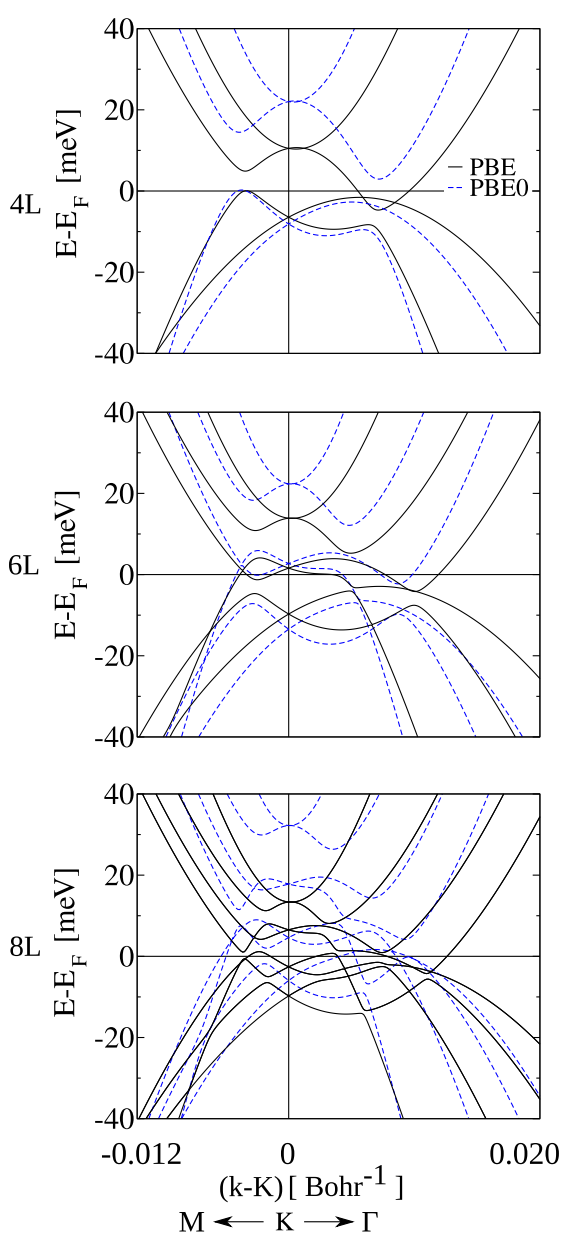
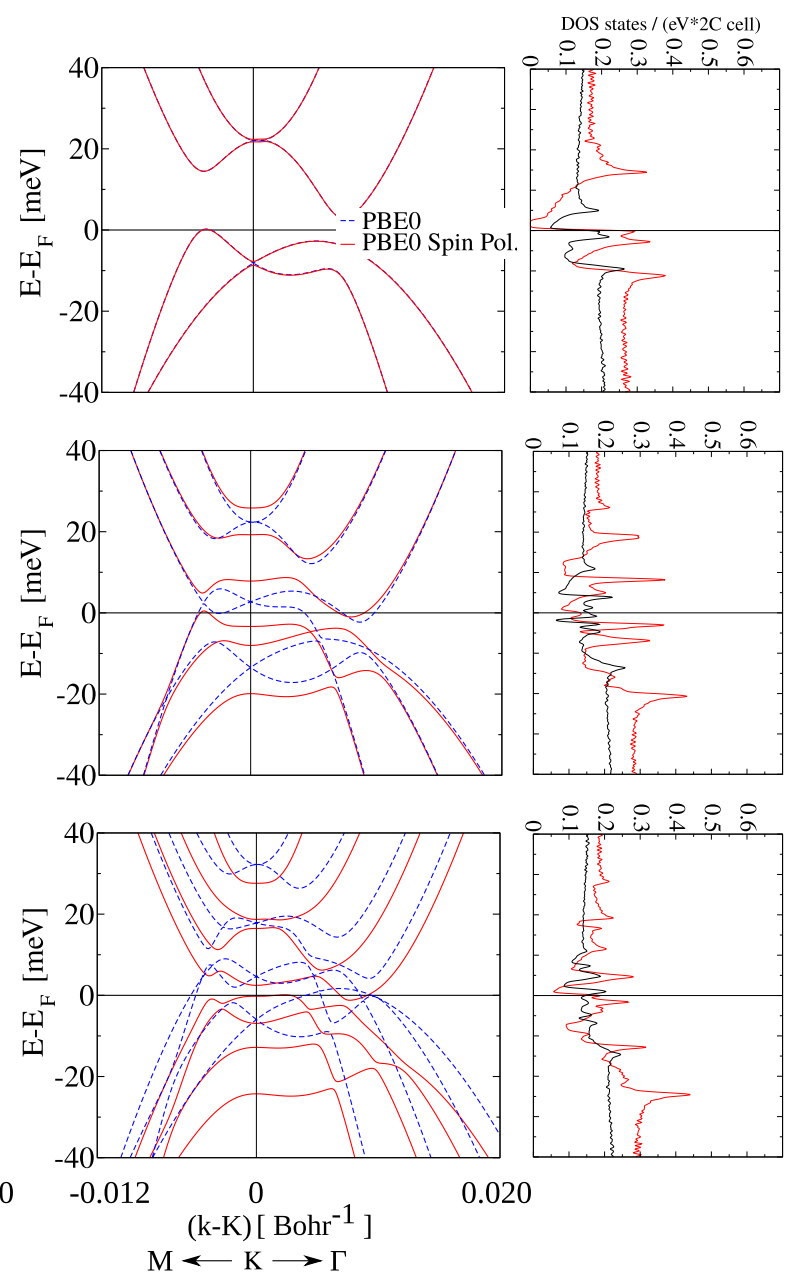

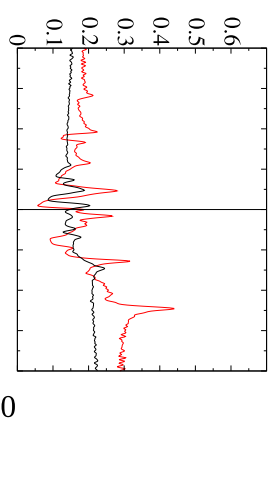

FIG. 7. Left panels: PBE versus PBE0 electronic structure for Bernal graphene multilayers with $N=4,6$, 8. Middle panels: PBE0 nonspin-polarized versus PBE0 spin-polarized electronic structures. The PBE0 magnetic and PBE nonmagnetic density of states are reported in the rightmost panels.

by atoms 4 and 5, namely atoms in the inner layers. This is very similar to the three-layer case, namely the character of the massive bands is due to atoms not involved in $\gamma_{1}$ hopping. Interestingly, there is an exchange in the band character close to the Fermi level at the top of the lowest energy bands along MK and at the bottom of the two highest energy bands. This character exchange is present also in a minimal model including only $\gamma_{0}$ and $\gamma_{1}$ [28], albeit the form of the bands in the PBE calculation, including all possible hopping processes and metallic screening, is substantially more complex. Unlike the three-layer system, in this case the introduction of the exact exchange does not change the composition of the bands. The only relevant change is the missing crossing bands along the MK high-symmetry direction.

\section{B. Electronic structure of thicker flakes}

\section{Odd number of layers}

The PBE and PBE0 electronic structures for $N=3,5,7$ are shown on the left panels of Fig. 6. We note that all structures are metallic, both in PBE and PBE0. In all cases, in a $40 \mathrm{meV}$ window from the Fermi level there are: (i) a gapped Dirac cone and (ii) $(N-1)$ paraboliclike bands. The latter are related to the massive bands in graphite and the separation between them is related to the $k_{z}$ dispersion of the electronic structure in graphite. The overlap between the Dirac band and the massive bands depends sensibly on the number of layers as well as on the exchange correlation functional used in the calculation. Both at the PBE and PBE0 level, the three- and seven-layer cases are pseudogapped close to the Fermi level (directly below or above), the pseudogap being larger for the PBE0. The pseudogap is larger for $N=3$ than for $N=7$, in qualitative disagreement with the gap inferred from transport data in Ref. [23].

The action of the exchange interaction, despite differing in details for different $N$, has some features common to all flakes composed of odd $N$. The first important point is that the slope of the Dirac cone close to $K$ as well as the slopes of the massive bands far from $K$ are increased by the exchange interaction. This corresponds to a well known [42-44] renormalization of the in-plane hopping matrix elements that is present both in graphene and graphite. Second, the exchange interaction tends to open gaps between bonding and nonbonding massive bands with opposite concavities. These two effects, combined with the conservation of the number of electrons, results in an upshift of the Dirac cone 
with respect to the massive bands. It is crucial to remark that this is exactly the opposite of the effect predicted in Ref. [28] based on self-consistent tight-binding calculations including empirical exchange interaction. The main reason is that in Ref. [28] the exchange interaction simply renormalizes $\gamma_{2}$, but not $\gamma_{1}$ that is kept at the experimental value in disagreement with what has been shown to occur in graphene. Including part of the renormalization in the $\gamma_{1}$ and varying the effect of the interaction on $\gamma_{2}$ only introduces an error and is equivalent to an ad hoc tuning of the position of the Dirac band. This underlines the need of performing explicit calculations of the exchange interaction beyond the tight-binding approach.

\section{Even number of layers}

In the case of an even number of layers, the electronic structure in a $40 \mathrm{meV}$ window from the Fermi level is composed of $N$ massive bands. As in the odd number of layer case, the exchange interaction tends to open gaps between the bonding and antibonding massive bands. However, here only the four layer becomes completely gapped (the gap being $3.2 \mathrm{meV}$ ) and insulating in PBE0 due to the removal of the weak band overlap present at the PBE level. For $N=6,8$, the flakes are metallic both in PBE and PBE0, however they develop small pseudogaps at the PBE0 level (1.8 and $1.6 \mathrm{meV}$ for $6 \mathrm{~L}$ and $8 \mathrm{~L}$, respectively). Even in this case, the magnitude and the behavior with thickness of the (pseudo) gaps disagree with experimental data given in Ref. [23].

\section{Magnetic states}

As several other carbon based systems such as rhombohedral stacked multilayer graphene $[11,12,16,17,20]$, twisted bilayer graphene [25,26,45], and diamond(111) [27] have been suggested to host magnetic states, it is meaningful to verify if magnetism can be stabilized in this system. For this reason we run spin-polarized calculations in PBE and PBE0. It is worthwhile to notice that in order to stabilize magnetism an ultradense grid of $k$ points needs to be used. Indeed, if the region close to the point $K$ and in a $20 \mathrm{meV}$ window from the Fermi level is not correctly sampled, the solution will always be nonmagnetic. Within PBE we never managed to stabilize a magnetic state. On the contrary, in PBE0 we stabilize a globally antiferromagnetic state. The starting magnetic state of our simulation is antiferromagnetic both globally and within each layer with antiferromagnetic coupling between layers. Only few flakes stabilize sizable magnetic moments, namely the $5 \mathrm{~L}, 6 \mathrm{~L}$, and $8 \mathrm{~L}$, all the other flakes had a negligible atomic magnetic moment $\left(\leqslant 10^{-4} \mu_{B}\right)$. All the magnetic ground states have three common features: first, all the magnetic states are globally antiferromagnetic (the bands are twofold degenerate in spin), second, each layer is ferrimagnetic, namely two close atoms have opposite spins but with a slightly different magnitude of the magnetic moments, and, finally, the magnitude of the spin drops significantly in going from the inner layers to the outer ones. The magnetic states are depicted in Fig. 8 and the magnetic moments reported in Table I. These results are consistent with a previous investigation performed on rhombohedral stacked graphene [20]: namely the presence of stable magnetic states in multilayer graphene systems. However, here there are two
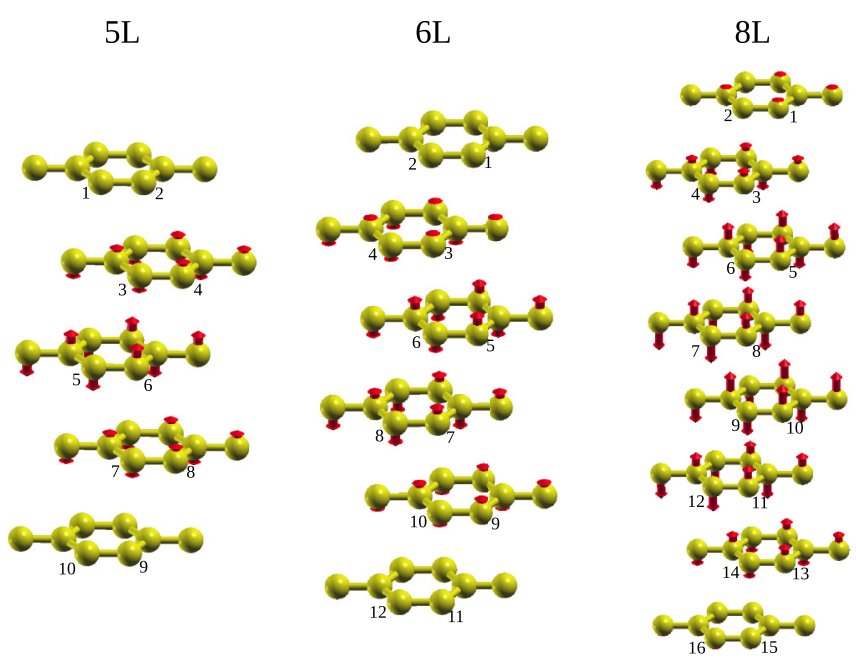

FIG. 8. Schematic representation of the magnetic ground state for $5 \mathrm{~L}, 6 \mathrm{~L}$, and $8 \mathrm{~L}$ systems, respectively, for Bernal graphene multilayers. The spins (as arrows) and atom numbering are reported.

main differences: (i) The magnetic moments increase going from the outer layers to the center (while in ABC graphene multilayers it is the opposite) and (ii) the magnitude of the magnetic moments is much smaller in the present case. The main reason for this difference holds in the nature of the character forming the massive bands. While in ABC graphene multilayers the massive bands are mostly formed by atoms in the outermost layers, in Bernal graphene multilayers the massive bands are formed by $\mathrm{B}$ atom types and the bonding ones mostly by $\mathrm{B}$ atoms in the innermost layers. Magnetism has some effects on the electronic structure of five, six, and eight layers, as shown in Figs. 6 and 7, mainly opening gaps and removing exact crossings at the $K$ highsymmetry point. However, not enough to make the system insulating. It slightly increases the pseudogaps found at the nonmagnetic PBE0 level.

\section{RESULTS FOR RHOMBOHEDRAL MULTILAYERS}

In our previous works $[14,17,20]$ carried out with the POB-TZVP basis set, we found that multilayer graphene with rhombohedral stacking has a magnetic ground state (at least for thicknesses up to 14 layers) with an ultraflat surface state. Given the lower accuracy of POB-TZVP basis sets in the $100 \mathrm{meV}$ energy region with respect to those developed in

TABLE I. The magnitude of the spin of each atom in units of $10^{-3} \mu_{B}$ are reported. Due to the symmetry of the magnetic states established, we report for $5 \mathrm{~L}$ spin up to $\mu_{6}$, since we have $\mu_{1}=\mu_{10}$, $\mu_{2}=\mu_{9}, \mu_{3}=\mu_{7}$, and $\mu_{4}=\mu_{8}$. For the systems with an even number of layers $(6 \mathrm{~L}$ and $8 \mathrm{~L}$ ) we found the following symmetry: $\mu_{i}=-\mu_{2 N-i+1}$ for $i=1, N$. Hence we report the spin up to $\mu_{N}$.

\begin{tabular}{ccccccccc}
\hline \hline$N$ & $\mu_{1}$ & $\mu_{2}$ & $\mu_{3}$ & $\mu_{4}$ & $\mu_{5}$ & $\mu_{6}$ & $\mu_{7}$ & $\mu_{8}$ \\
\hline 5 & 1.56 & -1.48 & 2.10 & -2.29 & 2.40 & -2.22 & & \\
6 & 0.71 & -0.65 & 1.08 & -1.26 & 1.70 & -1.47 & & \\
8 & 0.93 & -0.83 & 1.44 & -1.67 & 2.20 & -1.90 & 2.21 & -2.24 \\
\hline
\end{tabular}




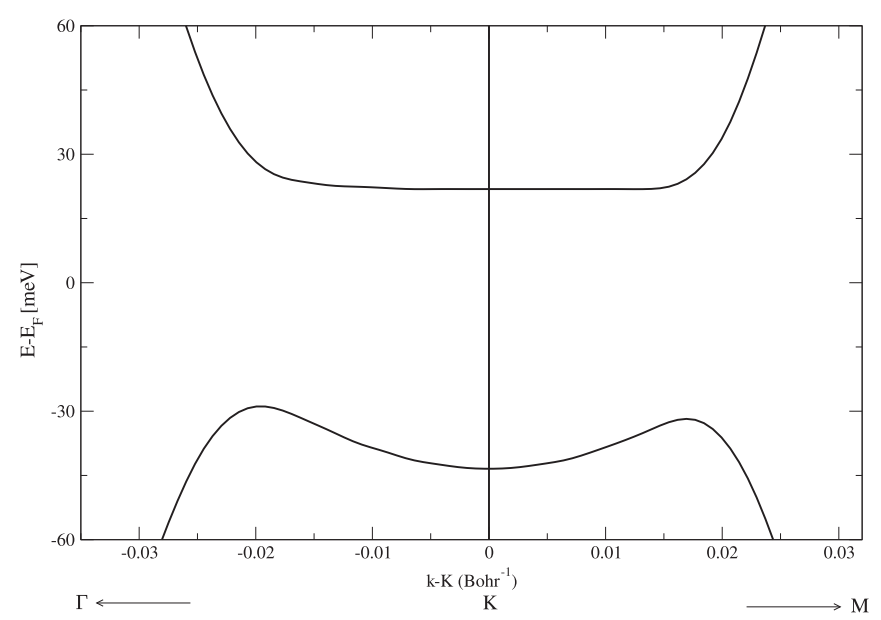

FIG. 9. Magnetic PBE0 electronic structure of six layers ABC graphene calculated using the most accurate $\mathrm{QZV}(\mathrm{P})$ basis set.

the current work, it is worthwhile to reevaluate electronic structure and magnetic properties in this energy region using the more accurate QZV $(\mathrm{P})$. This is the purpose of the current section. We consider 6 and 14 layers (see Figs. 9 and 10). Calculations on three layers were recently carried out by us in Ref. [13] using the QZV(P) finding small differences with the case of Refs. [14,20]. However, the situation could change in thicker samples as the main difference between the QZV(P) and POB-TZVP basis sets is the treatment of long distance hoppings.

\section{A. Six layers}

Within PBE0, the ground state of six layers ABC graphene is antiferromagnetic. Qualitatively and quantitatively, practically no differences are found for what concerns the magnetic state with respect to Ref. [20]. The ground state is the layer antiferromagnet, usually labeled LAF [11], with the largest magnetic moments concentrated in the outermost layers. The electronic structure, however, does show some differences. The indirect gap is of similar magnitude $(\approx 50 \mathrm{meV})$, but the dispersion and effective mass of the valence and conduction

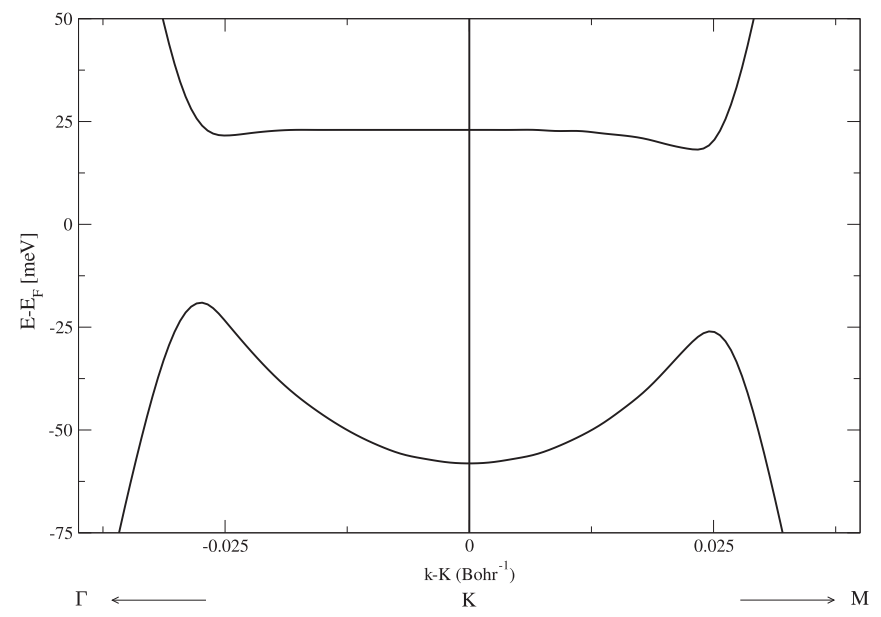

FIG. 10. Magnetic PBE0 electronic structure of 14 layers ABC graphene calculated using the most accurate QZV $(\mathrm{P})$ basis set.

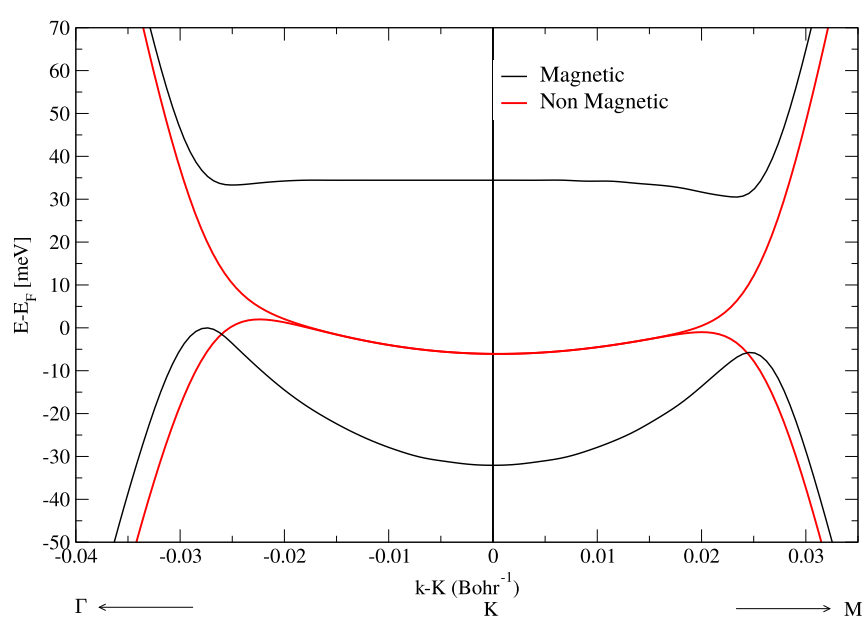

FIG. 11. Magnetic and non-magnetic PBE0 electronic structure of 14 layers rhombohedral stacked multilayer graphene calculated using the most accurate $\mathrm{QZV}(\mathrm{P})$ basis set. In order to directly compare with ARPES in Ref. [17], we reduce Fermi velocity by $16 \%$, as it is well known that PBE0 gives a too large exchange renormalization of the Fermi velocity with respect to GW in graphene [43]. To better compare the two calculations the Fermi level for the magnetic insulating case has been set at the valence band top.

bands depend substantially on the basis set used. In particular, by using the most accurate $\mathrm{QZV}(\mathrm{P})$, the dispersion of the top of the valence band from its maximum along $\Gamma K$ to the $K$ point is approximately $13 \mathrm{meV}$, while the less accurate POB-TZVP basis set leads to 7-8 $\mathrm{meV}$ dispersion, namely $\approx 35 \%-40 \%$ underestimation. On the contrary, the curvature of the bottom of the conduction band at $K$ is substantially smaller by using the more accurate QZV(P) basis set (the conduction band is almost flat ar $K$ ) with respect to the POBTZVP basis set.

\section{B. 14 layers and comparison with ARPES experiments}

The 14 layers calculation is important as the dispersion and curvature of the valence band close to the point $K$ have been used in ARPES [17] to identify the occurrence of a possible magnetic state. In experiments, the band dispersion of the valence band top, from its minimum at $K$ to the maximum along $\Gamma K$, was estimated to be $\approx 25 \mathrm{meV}$. As shown in Sec. II B, within the PBE0 approximation, in the nonmagnetic case and using the POB-TZVP basis set, the dispersion is smaller than $2 \mathrm{meV}$, but it increases to $\approx 8 \mathrm{meV}$ when the QZV(P) basis set is used. In the magnetic case, the band dispersion using the most accurate QZV (P) basis set is shown in Fig. 10 and is approximately $40 \mathrm{meV}$. This demonstrates that the curvature in the magnetic case is substantially enhanced with respect to the nonmagnetic case, in agreement with the results of Ref. [17]. In order to compare with experiments, some care is needed, as it is well known that for graphene the PBE0 exchange and correlation functional overestimates the Fermi velocity of approximately $16 \%$ [43]. By applying this reduction, the dispersion of the top of the valence band is $\approx 33 \mathrm{meV}$, not too far from the $25 \mathrm{meV}$ estimated in ARPES experiments [17]. The difference between the magnetic and nonmagnetic band structure is substantial, as shown in Fig. 11, 
and the ARPES results clearly show a better agreement with a magnetic electronic structure, as concluded in Ref. [17].

\section{CONCLUSIONS}

In this paper we study the electronic structure of multilayer graphene by means of hybrid functionals within a localized basis set approach. We show that basis sets normally considered as very accurate, such as the POB-TZVP one, fail substantially in predicting the electronic structure of multilayer graphene with Bernal stacking. For rhombohedral stacking, the error is much smaller, as the gap and magnetic state are essentially identical to those calculated with more extended basis sets, but the curvature and dispersion of the valence band top is substantially underestimated (the effect is negligible for trilayers but become important for thicker multilayers). We solved this problem by developing two new basis sets that perfectly reproduce the plane-waves calculations and lead to a very accurate description of the electronic structure in the $100 \mathrm{meV}$ range from the Fermi level.

With these new basis sets, we study the effects of exact exchange as included in the PBE0 functional on the electronic structure. For Bernal multilayers, we found that in all case (except five layers) the exchange interaction opens gaps (four layers) or pseudogaps (three, six, seven, and eight layers). However, both the size and the thickness dependence of the pseudogaps disagree with experiments [23]. For five, six, and eight layers, the ground state is found to be magnetic with very small magnetic moments of the order of $10^{-3} \mu_{B}$. These magnetic moments have very small effects on the nonmagnetic electronic structure. The magnetic state is such that the inner layers are weakly ferrimagnetic in the graphene plane and the surface layers have vanishing momenta within a globally antiferromagnetic state. Thus, at contrast with ABC multilayer graphene, the magnitude of the magnetic moments increases going from the outer layers to the center. Given that our results for both $\mathrm{ABA}$ and $\mathrm{ABC}$ [13] trilayers are practically indistinguishable from nonmagnetic $G W$ calculations in Ref. [10], we can infer that the screening of the exchange interaction is described satisfactorily by the hybrid functional. As the effect of magnetism on the band structure of ABstacked multilayers is nearly negligible, the $G W$ screening can be expected not to change as well. Therefore, the approach used in this work is close to an optimal mean-field theory both in the presence and in the absence of magnetism. Our results rule out a gap opening due to single-particle effects, including spin symmetry breaking, in perfect $A B$ stacked multilayers. Two alternative explanations for the experimentally observed insulating states are left. The first is that many-body effects beyond mean-field theory are responsible for the gap opening. A second possibility is that the gap is not intrinsic and is triggered by some kind of external interaction (small electric fields, residual doping, asymmetry in the samples along the $z$ axis, etc.). In order to shed some light on the effects of one of this possible external factors, we have performed SCF calculations at PBE0 level on the six-layer system using an external electric field (orthogonal to the graphene sheets) with variable intensity, in the absence of magnetism. A perpendicular electric field breaks the symmetry along the $z$ axis and can induce a gap opening in bilayers $[46,47]$ as well as ABCstacked multilayers [14]. However, as can be seen in Fig. 1 of the SM [33], the gap opening is absent also in this case. The main effect of the electric field is to make the bands more particle-hole symmetric. Even considering the possibility of magnetism coupled to the electric field, as shown in Fig. 2 of the SM [33], we were unable to stabilize a gap opening. More work is needed to clarify this issue.

Finally, we reevaluate the effect of more accurate basis sets for the case of 6 and 14 graphene multilayer with rhombohedral stacking. While the magnetic ground state properties and the gap are essentially unchanged with respect to previous calculations using less accurate basis sets [14,17,20], the dispersion of the valence band top (conduction band bottom) was underestimated (overestimated). The underestimation of the valence band top energy dispersion is larger in the nonmagnetic case, while it is smaller in the magnetic case. At odds with the Bernal case, in the rhombohedral multilayers the stabilization of larger magnetic moments and the favorable comparison with ARPES suggest that mean-field theory including the exchange interaction correctly describes the ground state properties of the system, in agreement with our previous results.

Our calculations represent a new benchmark for the theoretical description of the low energy physics graphene multilayers beyond standard density functional theory with semilocal kernels and will be a reference for future many-body calculations.

\section{ACKNOWLEDGMENTS}

We acknowledge support from the Graphene Flagship Core 2 Grant No. 785219 and Agence Nationale de la Recherche under references ANR-17-CE24-0030. This work was performed using HPC resources from GENCI, TGCC, CINES, IDRIS (Grant No. 2019-A0050901202 and The Grand Challenge Jean Zay).
[1] M. Koshino, New J. Phys. 15, 015010 (2013).

[2] B. Partoens and F. M. Peeters, Phys. Rev. B 74, 075404 (2006).

[3] M. Koshino and T. Ando, Solid State Commun. 149, 1123 (2009).

[4] M. Koshino and E. McCann, Phys. Rev. B 80, 165409 (2009).

[5] M. Koshino and T. Ando, Phys. Rev. B 76, 085425 (2007).

[6] H. Min and A. H. MacDonald, Prog. Theor. Phys. Suppl. 176, 227 (2008).

[7] S. Latil and L. Henrard, Phys. Rev. Lett. 97, 036803 (2006).
[8] M. Aoki and H. Amawashi, Solid State Commun. 142, 123 (2007).

[9] A. Sabashvili, S. Östlund, and M. Granath, Phys. Rev. B 88, 085439 (2013).

[10] M. G. Menezes, R. B. Capaz, and S. G. Louie, Phys. Rev. B 89 035431 (2014).

[11] Y. Lee, D. Tran, K. Myhro, J. Velasco, N. Gillgren, C. N. Lau, Y. Barlas, J. M. Poumirol, D. Smirnov, and F. Guinea, Nat Commun. 5, 5656 (2014). 
[12] K. Myhro, S. Che, Y. Shi, Y. Lee, K. Thilahar, K. Bleich, D. Smirnov, and C. N. Lau, 2D Mat. 5, 045013 (2018).

[13] Y. Lee, S. Che, J. Velasco Jr., D. Tran, J. Baima, F. Mauri, M. Calandra, M. Bockrath, and C. N. Lau, arXiv:1911.04450.

[14] J. Baima, F. Mauri, and M. Calandra, Phys. Rev. B 98, 075418 (2018).

[15] A. Kerelsky, C. Rubio-Verdú, L. Xian, D. M. Kennes, D. Halbertal, N. Finney, L. Song, S. Turkel, L. Wang, K. Watanabe, T. Taniguchi, J. Hone, C. Dean, D. Basov, A. Rubio, and A. N. Pasupathy, arXiv:1911.00007.

[16] Y. Henni, O. P. Collado, K. Nogajewski, M. R. Molas, G. Usaj, C. A. Balseiro, M. Orlita, M. Potemski, and C. Faugeras, Nano Lett. 16, 3710 (2016).

[17] H. Henck, J. Avila, Z. BenAziza, D. Pierucci, J. Baima, B. Pamuk, J. Chaste, D. Utt, M. Bartos, K. Nogajewski, B. A. Piot, M. Orlita, M. Potemski, M. Calandra, M. C. Asensio, F. Mauri, C. Faugeras, and A. Ouerghi, Phys. Rev. B 97, 245421 (2018).

[18] Y. Shi, S. Xu, Y. Yang, S. Slizovskiy, S. V. Morozov, S. K. Son, S. Ozdemir, C. Mullan, J. Barrier, J. Yin, A. I. Berdyugin, B. A. Piot, T. Taniguchi, K. Watanabe, V. I. Fal'ko, K. S. Novoselov, A. K. Geim, and M. Mishchenko, arXiv:1911.04565.

[19] A. Torche, F. Mauri, J. C. Charlier, and M. Calandra, Phys. Rev. Mater. 1, 041001 (2017).

[20] B. Pamuk, J. Baima, F. Mauri, and M. Calandra, Phys. Rev. B 95, 075422 (2017).

[21] F. Freitag, J. Trbovic, M. Weiss, and C. Schönenberger, Phys. Rev. Lett. 108, 076602 (2012).

[22] Y. Nam, D.-K. Ki, M. Koshino, E. McCann, and A. F. Morpurgo, 2D Mater. 3, 045014 (2016).

[23] Y. Nam, D.-K. Ki, D. Soler-Delgado, and A. F. Morpurgo, Science 362, 324 (2018).

[24] A. L. Grushina, D.-K. Ki, M. Koshino, A. A. L. Nicolet, C. Faugeras, E. McCann, M. Potemski, and A. F. Morpurgo, Nat. Commun. 6, 6419 (2015).

[25] Y. Cao, V. Fatemi, S. Fang, K. Watanabe, T. Taniguchi, E. Kaxiras, and P. Jarillo-Herrero, Nature (London) 556, 43 (2018).

[26] Y. Cao, V. Fatemi, A. Demir, S. Fang, S. L. Tomarken, J. Y. Luo, J. D. Sanchez-Yamagishi, K. Watanabe, T. Taniguchi, and E. Kaxiras, Nature (London) 556, 80 (2018).

[27] B. Pamuk and M. Calandra, Phys. Rev. B 99, 155303 (2019).
[28] M. Koshino, K. Sugisawa, and E. McCann, Phys. Rev. B 95, 235311 (2017).

[29] T. Stauber, P. Parida, M. Trushin, M. V. Ulybyshev, D. L. Boyda, and J. Schliemann, Phys. Rev Lett. 118, 266801 (2017).

[30] R. Dovesi, R. Orlando, A. Erba, C. M. Zicovich-Wilson, B. Civalleri, S. Casassa, L. Maschio, M. Ferrabone, M. De La Pierre, P. D'Arco et al., Int. J. Quantum Chem. 114, 1287 (2014).

[31] C. Adamo and V. Barone, J. Chem. Phys. 110, 6158 (1999).

[32] F. Weigend and R. Ahlrichs, Phys. Chem. Chem. Phys. 7, 3297 (2005).

[33] See Supplemental Material at http://link.aps.org/supplemental/ 10.1103/PhysRevB.101.165437 for all the basis sets used and the electronic bands for the $6 \mathrm{~L}$ system computed with different external electric fields.

[34] P. Giannozzi, S. Baroni, N. Bonini, M. Calandra, R. Car, C. Cavazzoni, D. Ceresoli, G. L. Chiarotti, M. Cococcioni, I. Dabo et al., J. Phys.: Condens. Matter 21, 395502 (2009).

[35] P. Giannozzi, O. Andreussi, T. Brumme, O. Bunau, M. B. Nardelli, M. Calandra, R. Car, C. Cavazzoni, D. Ceresoli, M. Cococcioni et al., J. Phys.: Condens. Matter 29, 465901 (2017).

[36] H. J. Monkhorst and J. D. Pack, Phys. Rev. B 13, 5188 (1976).

[37] M. F. Peintinger, D. V. Oliveira, and T. Bredow, J. Comput. Chem. 34, 451 (2013).

[38] https://www.basissetexchange.org.

[39] L. Daga, B. Civalleri, and L. Maschio, J. Chem. Theory Comput. (submitted).

[40] J. W. McClure, Phys. Rev. 108, 612 (1957).

[41] J. C. Slonczewski and P. R. Weiss, Phys. Rev. 109, 272 (1958).

[42] T. Ohta, T. Bostwick, T. Syller, K. Horn, and E. Rotenberg, Science 313, 951 (2006).

[43] S. Y. Zhou, G. H. Gweon, J. Graf, A. V. Fedorov, C. D. Spataru, R. D. Diehl, Y. Kopelevich, D. H. Lee, S. G. Louie, and A. Lanzara, Nat. Phys. 2, 595 (2006).

[44] S. Sugawara, T. Sato, S. Souma, T. Takahashi, and H. Suematsu, Phys. Rev. Lett. 98, 036801 (2007).

[45] L. A. Gonzalez-Arraga, J. L. Lado, F. Guinea, and P. San-Jose, Phys. Rev. Lett. 119, 107201 (2017).

[46] E. V. Castro, K. S. Novoselov, S. V. Morozov, N. M. R. Peres, J. M. B. dos Santos, J. Nilsson, F. Guinea, A. K. Geim, and A. H. Castro Neto, Phys. Rev. Lett. 99, 216802 (2007).

[47] P. Gava, M. Lazzeri, A. M. Saitta, and F. Mauri, Phys. Rev. B 79, 165431 (2009). 